\title{
Level-shift Modulation and Control of a Dual H-bridge Current Flow Controller in Meshed HVDC systems
}

\author{
Wei Liu, Jun Liang, C. E. Ugalde-Loo, Chuanyue Li, Gen Li, Peng Yang \\ Cardiff University, Cardiff, UK. \\ Liuw28@Cardiff.ac.uk
}

\begin{abstract}
The dual H-bridge current flow controller (CFC) has been proposed as a cost-effective solution to regulate current flows in a meshed high-voltage direct-current (HVDC) system. With an interline connection, this device may increase or decrease the magnitude and even reverse the directions of transmission line currents in a meshed de grid. However, it needs to be modulated in a different manner when its operating region changes, which makes its control more complex. This paper investigates a modulation method for the dual H-bridge CFC. The buck and boost regions of operation for the device are analyzed first. A level-shift modulation method based on pulse width modulation is proposed. This method ensures that only one bridge of the CFC is switching for each operating region, which simplifies the control of the device. In addition, the method ensures a smooth transition between the operating regions, which can enhance the reliability of the CFC. For completeness, the presented modulation and control methods are verified through time-domain simulations conducted in PSACD/EMTDC.
\end{abstract}

Keywords-Meshed HVDC system, current flow control, dual H-bridge CFC, level-shift modulation

\section{INTRODUCTION}

Voltage source converter (VSC) based HVDC technology exhibits important advantages over traditional line commutated converters (LCC) for the large-scale integration of renewable energy generation [1], [2]. The construction of multi-terminal dc (MTDC) grids based on VSC stations has been widely discussed in the literature as a means to enhance the flexibility and stability of transmission systems and further promote the system economic effectiveness [3]. However, there are still important obstacles preventing the wide-scale deployment of meshed MTDC grids. One of them is the effective regulation of current (or power) flow in a dc system, as this is dominated by the resistance of the transmission lines [4]. Therefore, currents cannot be directly controlled by the converters in the nodes, which may result in overloading of transmission lines.

CFC devices can be employed to effectively regulate line currents within a dc grid and to avoid transmission line overloading [5]. Several CFC topologies have been proposed in the open literature [6]-[8]. Inserting resistors (passive devices) in series with dc lines is a direct way to regulate the current. However, this solution incurs extra power losses [6]. An active ac/dc source could be also inserted into dc transmission lines [7], [8]. This way, power losses are reduced by exchanging power with a nearby ac grid. However, the large volume and high cost of the isolation transformer of an ac/dc source may limit the application of this solution in a practical HVDC system. To overcome the shortcomings previously discussed, several interline $\mathrm{dc} / \mathrm{dc}$ converters have been proposed to regulate the current flow [9]-[12]. The dual $\mathrm{H}$-bridge $\mathrm{CFC}$ is capable of achieving current flow regulation and does not require isolation transformers as it is powered within the de grid [13].

A number of modulation methods have been proposed for the dual H-bridge CFC [13]-[15]. In [13], a dual modulation technique is presented. By using two pulse-width modulation (PWM) signals, both the currents of the transmission lines and the voltage of the CFC capacitor can be controlled. However, this method incurs extra switching losses. A single modulation method is proposed in [15]. A single PWM signal is used to control the currents, which can reduce switching losses. However, all these methods rely on the precise detection of the current direction in the transmission lines to generate PWM signals. In a real application, control of the CFC may be lost during transient conditions. The transition between different operating regions is still a concern when the existing modulation methods are employed.

This paper presents a modulation method for dual $\mathrm{H}-$ bridge CFCs, termed level-shift modulation, where the CFC bridges are regulated by independent PWM signals. To avoid interactions between the signals, their carriers are shifted between each other. This brings the advantage that a single bridge is switched under a specific operating region. Additionally, the method avoids using instantaneous current signals in the transmission lines for the selection of the operating region of the $\mathrm{CFC}$, which reduces the influence of the current detection on the control of the device. This can ensure a smooth operation and reliability of the CFC. For completeness, the proposed modulation and control methods are verified through time-domain simulations conducted in PSCAD/EMTDC.

\section{Operating REgiOnS OF THE DUAL H-BRIDGE CFC}

A three-terminal VSC-based HVDC system, shown in Fig. 1 , is employed to analyze the performance of a dual $\mathrm{H}$-bridge CFC. The CFC is connected in Node 1 to regulate the currents between Line $a$ and Line $b$. The node current $I_{1}$ can be seen as constant since it is controlled by VSC1.

The basic topology of the device is shown in Fig. 2. It consists of two H-bridges ( $a$ and $b$ ) with paralleled capacitors. Since the switching pairs connected with Node 1 are in parallel with each other, the topology of the CFC can be simplified by merging the paralleled devices. This results in the simplified topology with three half-bridges as shown in Fig. 3. Such a topology has been adopted to illustrate the 


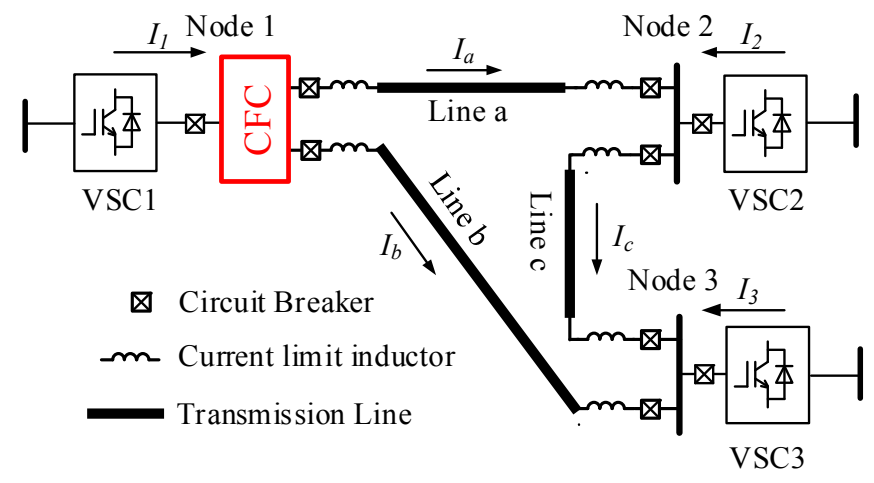

Fig. 1. Three-terminal VSC based HVDC system with dual H-bridge CFC.

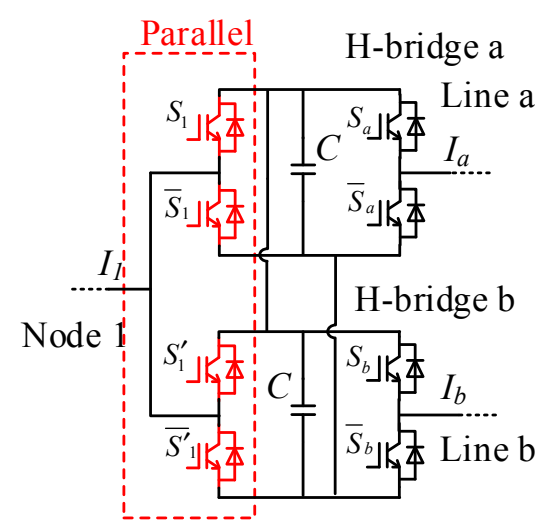

Fig. 2. Schematic of dual H-bridge CFC.

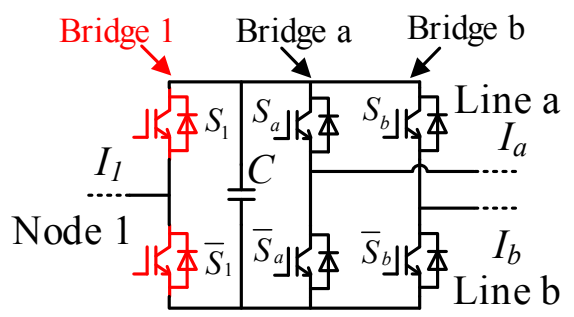

Merged

Fig. 3. Simplified topology of dual H-bridge CFC [6].

commutation process, which is demonstrated in Figs. 4 and 5. The switches in each bridge work complementarily. Based on the relationship between the input current $I_{1}$ and output currents $I_{a}$ and $I_{b}$, the operating regions of the CFC are classified as buck and boost regions.

\section{A. Buck Region}

The CFC operates in the buck region when Bridge 1 is modulated by a PWM signal. In this case, $S_{a}$ is kept in an 'on' state and $S_{b}$ is kept 'off', as shown in Fig. 4 . When $S_{1}$ is 'on', the capacitor is charged by $i_{c}=I_{1}-I_{a}$ (see Fig. 4(a)). Conversely, when $S_{1}$ is 'off', the capacitor is discharged by $i_{c}$ $=I_{a}$ (see Fig. 4(b)). To ensure energy balance of the capacitor, the average current (charging and discharging) flowing through the capacitor within a period of the PWM should be zero in the steady-state:

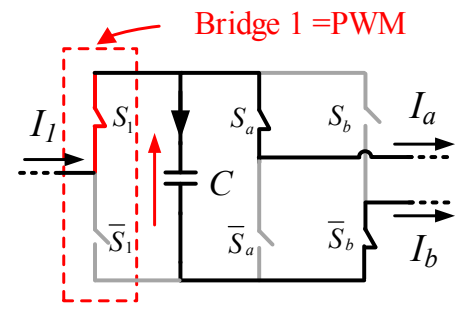

I: Charging $i_{c}=I_{1}-I_{a}$

(a)

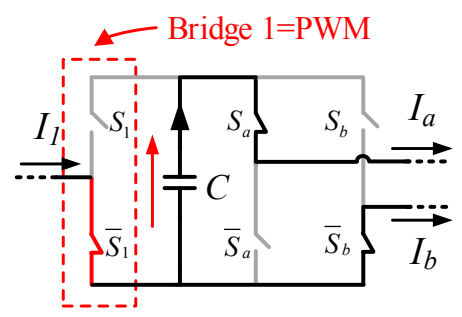

II: Dis charging $i_{c}=I_{a}$

(b)

Fig. 4. Buck region of dual H-bridge CFC. (a) Charging. (b) Discharging.

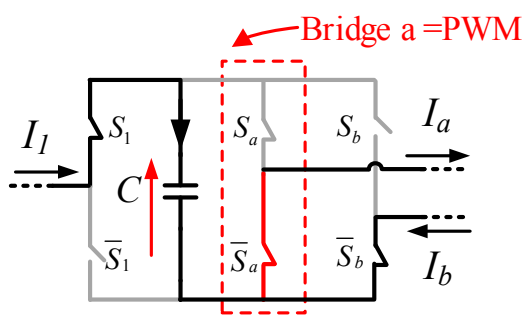

I: Charging $i_{c}=I_{a}$

(a)

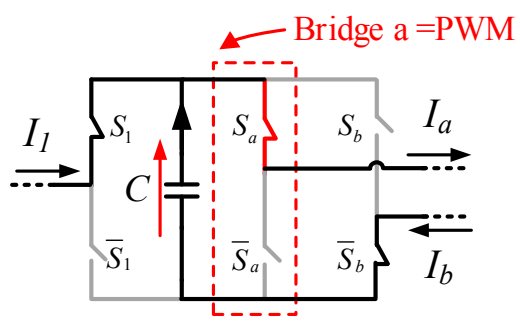

II: Dis charging $i_{c}=I_{a}-I_{1}$

(b)

Fig. 5. Boost region of dual H-bridge CFC. (a) Charging. (b) Discharging.

$$
\begin{gathered}
\bar{I}_{c}=\frac{1}{T} \int_{0}^{T} i_{c}(t) d t=\frac{\left(I_{1}-I_{a}\right) \times D T-I_{a}(1-D) T}{T}=0 \\
I_{a}=I_{1} D, I_{b}=I_{1}(1-D)
\end{gathered}
$$




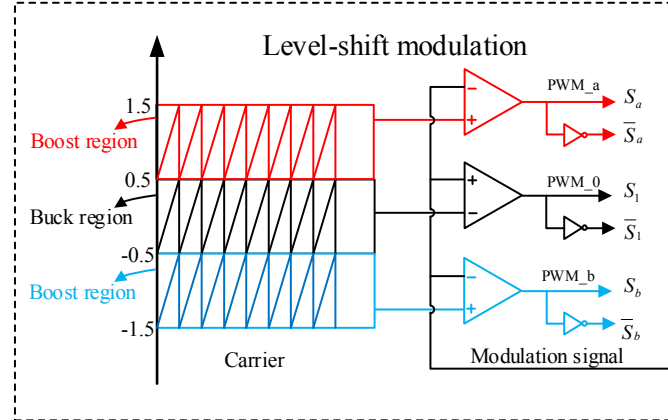

(a)

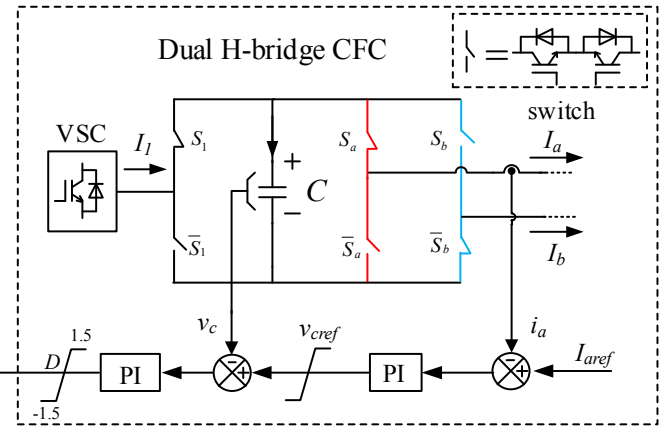

(b)

Fig. 6. Modulation and control strategy of the CFC using switches without free-wheeling paths. (a) Modulation method. (b) Dual-loop control of the CFC.

where $T$ is the period of the PWM and $D$ is the duty cycle of the PWM.

From (1), it can be seen that the currents in the transmission lines are regulated by $D$. As its value is always between 0 and 1 (i.e. $0<D<1$ ), line currents $I_{a}$ and $I_{b}$ will be smaller than the node current $I_{1}$ under the buck region. This operating mode can be used to balance the current flows between two transmission lines.

\section{B. Boost Region}

The device operates in the boost region when either Bridge $a$ or Bridge $b$ is modulated by a PWM signal. Fig. 5 illustrates the case for Bridge $a$, where $S_{1}$ is kept in an 'on' state and $S_{b}$ is kept 'off'. When $S_{a}$ is 'off', the capacitor is charged by $i_{c}=I_{a}$ (see Fig. 5(a)). When $S_{a}$ is 'on', the capacitor is discharged by $i_{c}=I_{a}-I_{1}$ (see Fig. 5(b)). In steadystate, the average current (charging and discharging) flowing through the capacitor should be zero within one period of the PWM. The following current expressions are derived:

$$
\begin{gathered}
\bar{I}_{c}=\frac{1}{T} \int_{0}^{T} i_{c}(t) d t=\frac{\left(I_{1}-I_{a}\right) \times(1-\mathrm{D}) T+I_{1} D T}{T}=0 \\
I_{a}=\frac{I_{1}}{1-D}, I_{b}=\frac{-D I_{1}}{1-D}
\end{gathered}
$$

It can be seen from (4) that the currents of the transmission lines are also regulated with the duty cycle $D$ $(0<D<1)$ as in the buck region. However, in this case line current $I_{a}$ will be higher than the node current $I_{1}$, while current $I_{b}$ will be reversed. The same result applies to $I_{b}$ when the PWM signal is applied instead to Bridge $b$.

\section{Summary}

The operating regions of the dual $\mathrm{H}$-bridge CFC in terms of PWM signals are summarized in Table I.

\begin{tabular}{|c|c|c|c|c|}
\hline \multirow{2}{*}{$\begin{array}{c}\text { Operating } \\
\text { Regions }\end{array}$} & \multicolumn{3}{|c|}{ Modulation signals } & Transmission line \\
\hline & Bridge a & Bridge 1 & Bridge $b$ & $I_{a}, I_{b}$ \\
\hline $\begin{array}{c}\text { Boost } \\
\text { Region }\end{array}$ & PWM_a & 1 & 0 & $I_{a}>I_{1}, I_{b}<0$ \\
\hline $\begin{array}{c}\text { Buck } \\
\text { Region }\end{array}$ & 1 & PWW_1 & 0 & $0<I_{a}<I_{1}, 0<I_{b}<I_{1}$ \\
\hline $\begin{array}{c}\text { Boost } \\
\text { Region }\end{array}$ & 1 & 0 & PWM_b & $I_{a}<0, I_{b}>I_{1}$ \\
\hline
\end{tabular}

TABLE I. OPERATION REgIONS OF THE DuAl H-BRIDGE CFC
From Table I, it can be seen that one operation region can be obtained by separately applying one PWM signal to a specific bridge. The magnitude of the currents in the transmission lines can be increased or decreased and their direction even reversed by the dual H-bridge CFC.

\section{LEVEL-SHIFT MODULATION AND CONTROL METHOD}

The operating regions of the CFC have been analyzed based on the simplified topology in Section II. According to the operating characteristics of the dual H-bridge CFC, levelshift modulation methods are presented in this section to simplify the control of the device.

\section{A. Level-shift modulation}

As shown in Fig. 6(a), the three carriers are shifted to avoid interactions with each other. Each carrier is used to generate an independent PWM signal. This modulation method ensures that only one bridge is regulated during the operation of the CFC. The dual-loop control for the CFC is shown in Fig. 6(b). The current in one transmission line (e.g. $\left.I_{a}\right)$ is fed back to the outer loop of the controller. The outer loop generates a voltage reference for the inner control loop to regulate the capacitor voltage.

It should be emphasized that this modulation and control method is suitable for CFC devices that employ switches without a free-wheeling path, as shown in Fig. 6(b). The capacitor voltage can have both a positive and a negative polarity, which is simple with regards to the control method. However, this kind of topology would require a precise detection of the current direction during switch commutation processes for a real application, which could damage the device if the current directions are wrongly detected.

A CFC employing switches with free-wheeling paths can overcome the above shortcoming. This is shown in Fig. 7(b). However, the free-wheeling diode can be seen as in parallel with the capacitor, which means that the capacitor voltage can only be positive. Additional control methods should be added when a negative capacitor voltage is required.

\section{B. Modified level-shift modulation}

A modified level-shift modulation method (see Fig. 7(a)) which employs switches with free-wheeling paths is proposed (see Fig. 7(b)). Two operating modes are considered for the carriers. The reference for the inner voltage control loop $v_{\text {cref }}$ is used to select a specific mode. When $v_{\text {cref }}$ 


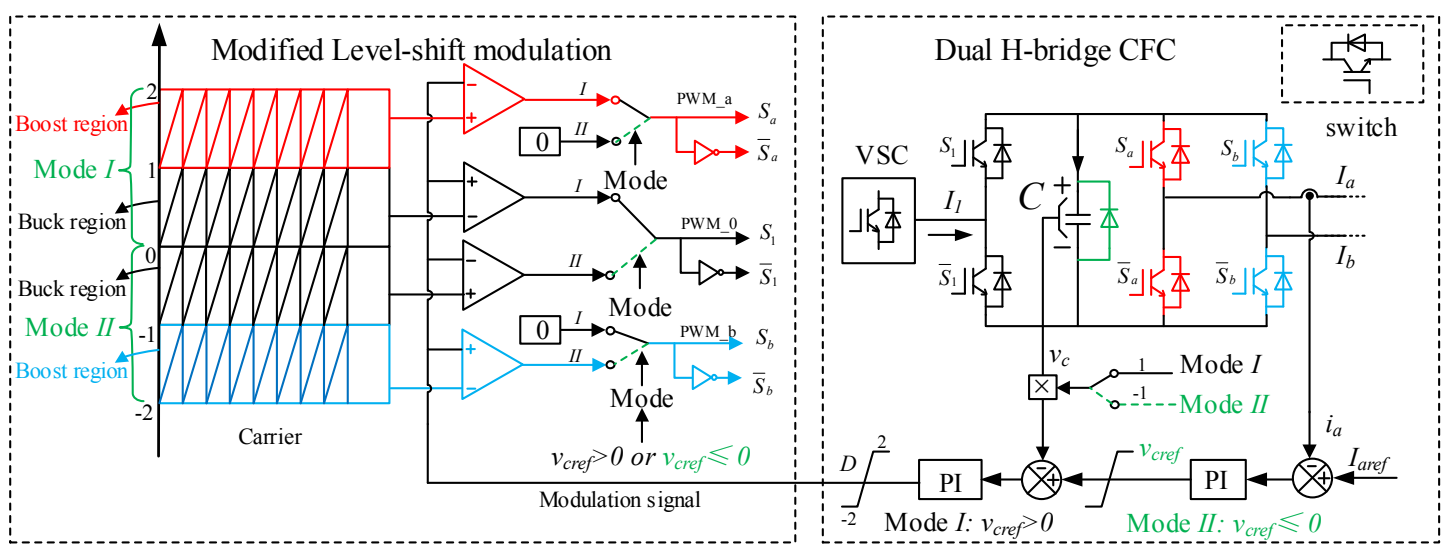

(a)

(b)

Fig. 7. Modulation and control strategy of the CFC using switches with free-wheeling path (a) Modulation method. (b) Dual-loop Control of the CFC.

$\geq 0$, the CFC is controlled as Mode I in Fig. 7. When $v_{\text {cref }}<0$, the positions of Lines $a$ and $b$ are exchanged (Mode II). This avoids using the current direction of the transmission lines to select the mode. With the mode transition from I to II, an equivalent negative capacitor voltage is obtained.

The modified level-shift modulation method brings the following advantages: 1) free-wheeling paths ensure a safe commutation, and 2) there is no need to use the line current directions to distribute PWM signals. Since the transition from one operating region to another is determined by signal $v_{\text {cref }}$ only, this method can ensure a smooth operation and reliability of the CFC device.

\section{Simulation ReSUlts}

An HVDC system (as Fig. 1) with a dual H-bridge CFC has been built in PSCAD/EMTDC to verify the presented modulation and control methods. Average models of the VSCs are used to simplify the system and to focus on the analysis of the CFC. The transmission lines are modeled as inductors and resistors. The system parameters are given in Table II. The switching frequency of the CFC is $1 \mathrm{kHz}$ and the capacitor in the CFC has a capacitance of $20 \mathrm{mF}$. In a real project, the switching frequency of the device would be selected based on the capability of the semiconductor device and the capacitor should be selected based the switching frequency accordingly. The node current $I_{1}$ is regulated to 1 $\mathrm{kA}$ by VSC1. $I_{a}$ is fed back to the CFC as a control target.

The simulation results based on the modified level-shift modulation method are shown in Fig. 8. Node current $I_{1}$ and currents $I_{a}$ and $I_{b}$ are given in Fig. 8(a).

TABLE II: SYSTEM PARAMETERS

\begin{tabular}{|c|c|}
\hline Parameters & Value \\
\hline Rated DC voltage & $500 \mathrm{kV}$ \\
\hline Rated power of VSC1 & $500 \mathrm{kVA}$ \\
\hline Rated power of VSC2 & $150 \mathrm{kVA}$ \\
\hline Rated power of VSC3 & $700 \mathrm{kVA}$ \\
\hline Inductor/ Resistor of Line a & $180 \mathrm{mH} / 2 \Omega$ \\
\hline Inductor/ Resistor of Line $\mathrm{b}$ & $150 \mathrm{mH} / 1 \Omega$ \\
\hline Inductor/ Resistor of Line c & $100 \mathrm{mH} / 0.2 \Omega$ \\
\hline Capacitor of the CFC & $20 \mathrm{mH}$ \\
\hline Switching frequency of CFC & $1 \mathrm{kHz}$ \\
\hline Rated voltage of CFC & $8 \mathrm{kV}$ \\
\hline
\end{tabular}

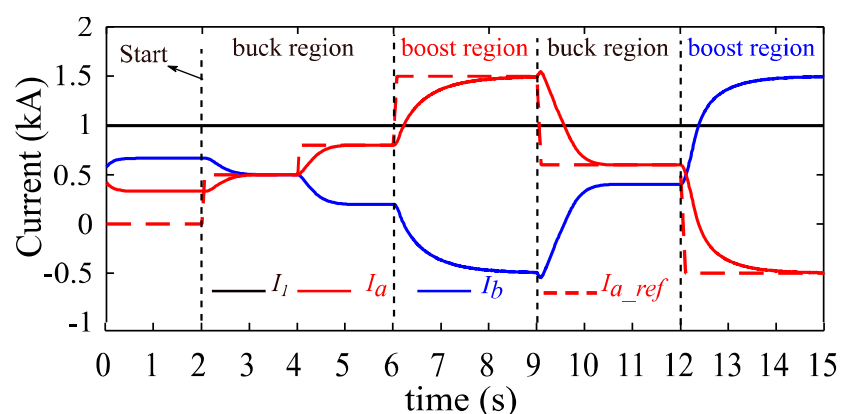

(a)

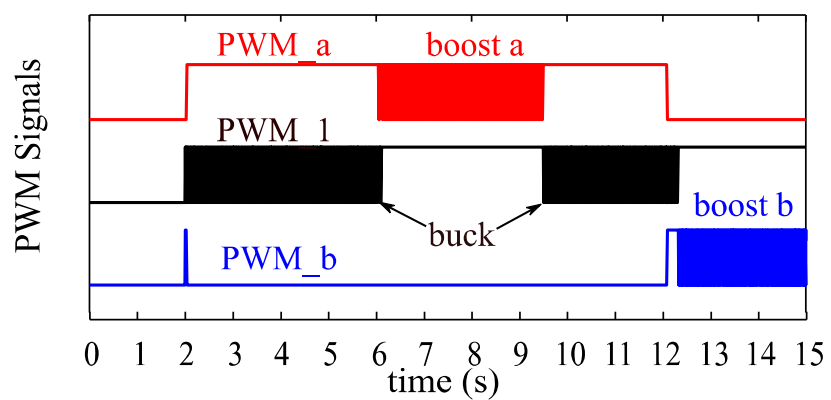

(b)

Fig. 8. Simulation results of the function of CFC in a mashed HVDC system (a) Transmission line currents. (b) PWM signals.

The CFC starts operating at $t=2 \mathrm{~s}$. It can be observed that $I_{a}$ follows properly its reference (red dashed line in Fig. 8(a)). As the value of reference $I_{a_{-} \text {ref }}$ changes, the CFC operates both in the buck region (between 2-6 and 9-12 s) and boost regions (between 6-9 and 12-15 s). It can be observed that a smooth transition between operating regions is achieved. Fig. 8(b) shows the PWM signals used to regulate the bridges of the CFC. It can be seen that only a single bridge is regulated by one PWM signal for each operating region, which is consistent with the analysis presented in the previous section.

\section{CONCLUSIONS}

A CFC is a device capable of achieving effective power flow regulation in a meshed MTDC grid. In this paper, the operating regions of a dual $\mathrm{H}$-bridge $\mathrm{CFC}$ have been analyzed and classified as buck and boost regions. It has been shown that each region can be controlled independently using a single bridge with a suitable PWM signal. These characteristics are exploited by the level-shift modulation 
method presented in this paper, where each bridge (linked to a single operating region) is regulated by an independent PWM signal without causing interactions with the modulation signal used for another bridge.

Suitable topologies to implement the level-shift modulation method using switches with and without freewheeling paths have been also investigated. A modified level-shift modulation method is proposed when switches with free-wheeling paths are employed, which simplifies the control strategy of the CFC device and enhances its reliability. For completeness, the proposed modulation methods with their corresponding control schemes have been verified via time-domain simulations conducted in PSCAD/EMTDC.

\section{ACKNOWLEDGMENT}

The authors gratefully acknowledge the funding from the project "Innovative Tools for Offshore Wind \& DC Grids (InnoDC)". This project has received funding from the European Union's Horizon 2020 Research and Innovation Program under the Marie Sklodowska-Curie grant agreement no. 765585 .

\section{REFERENCES}

[1] D. Jovcic and K. Ahmed, High-Voltage Direct Current Transmission: Converter System and DC Grids. Hoboken, NJ, USA: Wiley, 2015.

[2] G. Li, W. Liu, T. Joseph, J. Liang, T. An, J. Lu, M. Szechtman, B. Andersen and Q. Zhuang, "Control Strategies of Full-Voltage to HalfVoltage Operation for LCC and Hybrid LCC/MMC based UHVDC Systems," Energies, vol. 12, no. 4, Feb. 2019.

[3] G. Li, C. Li, D. Van Hertem, "HVDC technology overview," in HVDC Grids: For Offshore and Supergrid of the Future, Hoboken, NJ, USA:Wiley, pp. 45-76, 2016.

[4] K. Rouzbehi, A. Miranian, A. Luna and P. Rodriguez, "Towards fully controllable multi-terminal DC grids using flexible DC transmission systems," in IEEE Energy Conversion Congress and Exposition (ECCE), 2014, pp. 1-5.
[5] E. Veilleux and B. Ooi, "Power flow analysis in multi-terminal HVDC grid," in Power Systems Conf. and Expo. (PSCE), pp. 1-7, 2011.

[6] C. Barker and R. Whitehouse, "A current flow controller for use in HVDC grids," in IET Int. Conf. on AC and DC Power Trans. (ACDC), 2012, pp. 1-5.

[7] Q. Mu, J. Liang, Y. Li and X. Zhou, "Power flow control devices in DC grids," in Power and Energy Society General Meeting IEEE, 2012, pp.1-7.

[8] D. Jovcic, M. Hajian, H. Zhang and G. Asplund, "Power flow control in dc transmission grids using mechanical and semiconductor based dc/dc devices," in Proc. IET Int. Conf. AC/DC Power Trans., 2012, pp. $1-6$.

[9] S. Balasubramaniam, C. E. Ugalde-Loo, J. Liang, T. Joseph, R. King and A. Adamczyk, "Experimental Validation of Dual H-Bridge Current Flow Controllers for Meshed HVdc Grids," IEEE Trans. Power Del. vol. 33, no. 1, pp. 381-392, Sep. 2017.

[10] Sau-Bassols, E. Prieto-Araujo, O. Gomis-Bellmunt and F. Hassan, "Selective Operation of Distributed Current Flow Controller Devices for Meshed HVDC Grids", IEEE Trans. Power Del., vol. 34, no. 1, pp. 107-118, Feb. 2019.

[11] W. Chen, X. Zhu, L. Yao, G. Ning, Y. Li, Z. Wang, W. Gu and X. Qu, "A Novel Interline DC Power-Flow Controller (IDCPFC) for Meshed HVDC Grids," IEEE Trans. Power Del. vol. 31, issue 4, pp. 17191727, Aug. 2016.

[12] J. Sau-Bassols, E. Prieto-Araujo, O. Gomis-Bellmunt and F. Hassan, "Series interline DC/DC Current Flow Controller for meshed HVDC grids," IEEE Trans. Power Del. vol. 33, issue 2, pp. 881-891, Apr 2017.

[13] F. Hassan, R. King, R. Whitehouse and C. Barker, "Double modulation control (DMC) for dual H-bridge current flow controller (2B-CFC)," in 17th European Conf. on Power Electron. and Applications (EPE'15 ECCE-Europe), Geneva, 2015, pp. 1-9.

[14] N. Deng, P. Wang, X. Zhang, G. Tang and J. Cao, "A DC current flow controller for meshed modular multilevel converter multi-terminal HVDC grids," CSEE J. Power Energy Syst., vol. 1, no. 1, pp. 43-51, Mar. 2015.

[15] S. Balasubramaniam, J. Liang and C.E. Ugalde-Loo, "Control, dynamics and operation of a dual H-bridge current flow controller," in IEEE Energy Conversion Congress and Exposition (ECCE), Montreal, 2015, pp. 1-8. 